\title{
Clinical predictors of post-septal disease in peri-orbital cellulitis
}

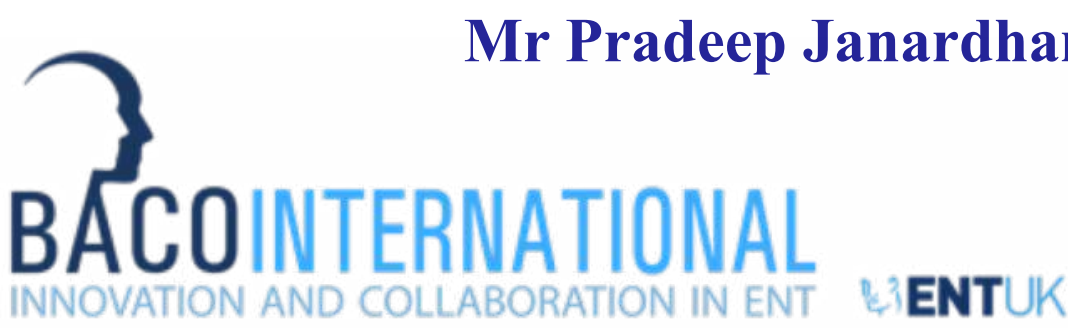

\author{
Institution \\ 1. New Cross Hospital, The Royal Wolverhampton NHS Trust, WV10 0QP \\ 2. Russells Hall Hospital, The Dudley Group of Hospitals, DY1 2HQ
}

\section{Background:}

Periorbital cellulitis is a well-recognised complication of sinus disease comprising of pre-septal and post-septal disease with the latter carrying higher morbidity and mortality. Urgent surgical intervention by otolaryngologists is often necessary for post-septal disease whereas pre-septal disease can normally be managed with antibiotic therapy.

It is thus important to distinguish the locus of disease early and when diagnostic uncertainty exists CT imaging may be required, the considerable radiation burden of this investigation needs to be

justified.

\section{Objectives:}

To investigate the predictive value of presenting clinical features and biochemical markers in identifying post-septal orbital disease.

Establish criteria for CT scanning in patients with periorbital cellulitis.

\section{Methods:}

Retrospective analysis of 51 consecutive patients treated for periorbital cellulitis over a 2-year period.

Data on clinical presentation were analysed using Statistical Package for the Social Sciences (SPSS20).

\section{Data domains:}

- Patient demographics

- Age

- Gender

- Clinical signs

- Eye signs

- Temperature
- Biochemical markers

- wcc

$$
\text { - CRP }
$$

- Microbiology

- blood cultures

- swab cultures

\section{Results:}

- 51 patients were identified in total

- Post-septal disease was identified in $18 \%(n=9)$

- 14 patients $(27 \%)$ had one or more red flag eye signs

- Sensitivity $=100 \%$

- Specificity $=88 \%$

- $\mathrm{NPV}=100 \%$

post septal disease

- There was no significant correlation between final diagnosis and any other clinical marker (Table 1).

Table 1: Clinical Predictors

\begin{tabular}{|c|c|c|}
\hline Factor impacting diagnosis & Significance & Test used \\
\hline Number of red flag eye signs & $\begin{array}{l}\mathrm{P}<0.001 \text { (significant) } \\
\mathrm{P}<0.001 \text { (significant) }\end{array}$ & $\begin{array}{l}\text { Pearson's R } \\
\text { Kruskal Wallis }\end{array}$ \\
\hline Type of red flag eye signs & $\begin{array}{l}\mathrm{P}<0.001 \text { (significant) } \\
\mathrm{P}<0.001 \text { (significant) }\end{array}$ & $\begin{array}{l}\text { Chi-squared } \\
\text { Simple logistic regression }\end{array}$ \\
\hline Sinogenic symptoms & $\begin{array}{l}\mathrm{P}=0.474 \text { (insignificant) } \\
\mathrm{P}=0.167 \text { (insignificant) }\end{array}$ & $\begin{array}{l}\text { Chi-squared } \\
\text { Simple logistic regression }\end{array}$ \\
\hline Febrile on admission & $\begin{array}{l}\mathrm{P}=0.502 \text { (insignificant) } \\
\mathrm{P}=0.240 \text { (insignificant) }\end{array}$ & $\begin{array}{l}\text { Chi-squared } \\
\text { Simple logistic regression }\end{array}$ \\
\hline White cell count & $\begin{array}{l}\mathrm{P}=0.073 \text { (insignificant) } \\
\mathrm{P}=0.086 \text { (insignificant) }\end{array}$ & $\begin{array}{l}\text { One way ANOVA } \\
\text { Simple linear regression }\end{array}$ \\
\hline C-Reactive Protein & $\begin{array}{l}P=0.366 \text { (insignificant) } \\
P=0.202 \text { (insignificant) }\end{array}$ & $\begin{array}{l}\text { One way ANOVA } \\
\text { Simple linear regression }\end{array}$ \\
\hline
\end{tabular}

\section{Discussion:}

Goldman et al. found that local ocular symptoms' (especially lid oedema) and pyrexia were the only significant predictors for admission in children with periorbital cellulitis.

Rudloe et al. identified that patients with proptosis, limitation of extra-ocular movements and neutrophilia are at higher risk of developing subperiosteal abscesses in periorbital cellulitis. They recommend CT scanning in this group of patients.

Our study corroborates these findings and adds more robust recommendations.

\section{Conclusion}

Eye signs are the only reliable clinical predictor for defining disease severity of periorbital cellulitis.

In the absence of eye signs CT imaging may not be justified. 